\title{
Existence of positive solutions for nonlinear $m$ - point boundary value problems on time scales
}

Junfang Zhao ${ }^{1 *}$, Hairong Lian ${ }^{1}$ and Weigao $\mathrm{Ge}^{2}$

\author{
* Correspondence: \\ zhao_junfang@163.com \\ ${ }^{1}$ School of Science, China \\ University of Geosciences, Beijing \\ 100083, P.R. China \\ Full list of author information is \\ available at the end of the article
}

\begin{abstract}
In this article, we study the following $m$-point boundary value problem on time scales,

$$
\left\{\begin{array}{l}
\left(\phi_{p}\left(u^{\Delta}(t)\right)\right)^{\nabla}+h(t) f(t, u(t))=0, \quad t \in(0, T) \mathbb{\pi}, \\
u(0)-\delta u^{\Delta}(0)=\sum_{i=1}^{m-2} \beta_{i} u^{\Delta}\left(\xi_{i}\right), \quad u^{\Delta}(T)=0,
\end{array}\right.
$$

where $\mathbb{T}$ is a time scale such that $0, T \in \mathbb{T}, \delta, \beta_{i}>0, i=1, \ldots, m-2$, $\varphi_{p}(s)=|s|^{p-2} s, p>1, h \in C_{l d}((0, T),(0,+\infty))$, and $f \in C([0,+\infty),(0,+\infty))$, $0<\xi_{1}<\xi_{2}<\cdots<\xi_{m-2}<T \in \mathbb{T}$. By using several well-known fixed point theorems in a cone, the existence of at least one, two, or three positive solutions are obtained. Examples are also given in this article.

AMS Subject Classification: 34B10; 34B18; 39A10.
\end{abstract}

Keywords: positive solutions, cone, multi-point, boundary value problem, time scale

\section{Introduction}

The study of dynamic equations on time scales goes back to its founder Hilger [1], and is a new area of still theoretical exploration in mathematics. Motivating the subject is the notion that dynamic equations on time scales can build bridges between continuous and discrete mathematics. Further, the study of time scales has led to several important applications, e.g., in the study of insect population models, neural networks, heat transfer, epidemic models, etc. [2].

Multipoint boundary value problems of ordinary differential equations (BVPs for short) arise in a variety of different areas of applied mathematics and physics. For example, the vibrations of a guy wire of a uniform cross section and composed of $N$ parts of different densities can be set up as a multi-point boundary value problem [3]. Many problems in the theory of elastic stability can be handled by the method of multi-point problems [4]. Small size bridges are often designed with two supported points, which leads into a standard two-point boundary value condition and large size bridges are sometimes contrived with multi-point supports, which corresponds to a multi-point boundary value condition [5]. The study of multi-point BVPs for linear second-order ordinary differential equations was initiated by Il'in and Moiseev [6]. Since then many authors have studied more general nonlinear multi-point BVPs, and

(c) 2012 Zhao et al; licensee Springer. This is an Open Access article distributed under the terms of the Creative Commons Attribution License (http://creativecommons.org/licenses/by/2.0), which permits unrestricted use, distribution, and reproduction in any medium, provided the original work is properly cited. 
the multi-point BVP on time scales can be seen as a generalization of that in ordinary differential equations.

Recently, the existence and multiplicity of positive solutions for nonlinear differential equations on time scales have been studied by some authors [7-11], and there has been some merging of existence of positive solutions to BVPs with $p$-Laplacian on time scales [12-19].

He [20] studied

$$
\left(\phi_{p}\left(u^{\Delta}(t)\right)\right)^{\nabla}+a(t) f(t)=0, \quad t \in(0, T)_{\mathbb{V}},
$$

subject to one of the following boundary conditions

$$
\left\{\begin{array}{l}
u(0)-B_{0}\left(u^{\Delta}(\eta)\right)=0, \quad u^{\Delta}(T)=0, \\
u^{\Delta}(0)=0, \quad u(T)-B_{1}\left(u^{\Delta}(\eta)\right)=0,
\end{array}\right.
$$

where $\eta \in(0, T) \cap \mathbb{T}$. By using a double fixed-point theorem, the authors get the existence of at least two positive solutions to BVP (1.1) and (1.2).

Anderson [21] studied

$$
-u^{\Delta^{\nabla}}(t)=\eta a(t) f(u(t)), \quad t \in\left(t_{1}, t_{n}\right) \pi,
$$

subject to one of the following boundary conditions

$$
\begin{aligned}
& u\left(t_{1}\right)=\sum_{i=2}^{n-1} \alpha_{i} u\left(t_{i}\right), \quad u^{\Delta}\left(t_{n}\right)=0, \\
& u^{\Delta}\left(t_{1}\right)=0, \quad u\left(t_{n}\right)=\sum_{i=2}^{n-1} \alpha_{i} u\left(t_{i}\right),
\end{aligned}
$$

by using a functional-type cone expansion-compression fixed-point theorem, the author gets the existence of at least one positive solution to BVP (1.3), (1.4) and BVP (1.3), (1.5).

However, to the best of the authors' knowledge, up to now, there are few articles concerned with the existence of $m$-point boundary value problem with $p$-Laplacian on time scales. So, in this article, we try to fill this gap. Motivated by the article mentioned above, in this article, we consider the following $m$-point BVP with one-dimensional $p$-Laplacian,

$$
\left\{\begin{array}{l}
\left(\phi_{p}\left(u^{\Delta}(t)\right)\right)^{\nabla}+h(t) f(t, u(t))=0, \quad t \in(0, T)_{\mathbb{W}}, \\
u(0)-\delta u^{\Delta}(0)=\sum_{i=1}^{m-2} \beta_{i} u^{\Delta}\left(\xi_{i}\right), \quad u^{\Delta}(T)=0,
\end{array}\right.
$$

where $\varphi_{p}(s)=|s|^{p-2} s, p>1, h \in C_{l d}((0, T),(0,+\infty)), 0<\xi_{1}<\xi_{2}<\cdots<\xi_{m-2}<T \in \mathbb{T}$. $\delta, \beta_{i}>0, i=1, \ldots, m-2$.

We will assume throughout

(S1) $h \in \mathrm{C}_{l d}((0, T),[0, \infty))$ such that $\int_{0}^{T} h(s) \nabla s<\infty$;

(S2) $f \in C([0, \infty),(0, \infty)), f \otimes 0$ on $f \not \equiv 0$ on $[0, T]_{\mathbb{T}}$;

(S3) By $\varphi_{q}$ we denote the inverse to $\varphi_{p}$, where $\frac{1}{p}+\frac{1}{q}=1$;

(S4) By $t \in[a, b]$ we mean that $t \in[a, b] \cap \mathbb{T}$, where $0 \leq a \leq b \leq T$. 


\section{Preliminaries}

In this section, we will give some background materials on time scales.

Definition 2.1. [7,22] For $t<\sup \mathbb{T}$ and $t>\inf \mathbb{T}$, define the forward jump operator $\sigma$ and the backward jump operator $\rho$, respectively,

$$
\sigma(t)=\inf \{\tau \in \mathbb{T} \mid \tau>t\} \in \mathbb{T}, \quad \rho(r)=\sup \{\tau \in \mathbb{T} \mid \tau<r\} \in \mathbb{T}
$$

for all $r, t \in \mathbb{T}$. If $\sigma(t)>t, t$ is said to be right scattered, and if $\rho(r)<r, r$ is said to be left scattered. If $\sigma(t)=t, t$ is said to be right dense, and if $\rho(r)=r, r$ is said to be left dense. If $\mathbb{T}$ has a right scattered minimum $m$, define $\mathbb{T}_{\kappa}=\mathbb{T}-\{m\}$; Otherwise set $\mathbb{T}_{\kappa}=\mathbb{T}$. The backward graininess $\mu_{b}: \mathbb{T}_{\kappa} \rightarrow \mathbb{R}_{0}^{+}$is defined by

$$
\mu_{b}(t)=t-\rho(t) .
$$

If $\mathbb{T}$ has a left scattered maximum $M$, define $\mathbb{T}^{\kappa}=\mathbb{T}-\{M\}$; Otherwise set $\mathbb{T}^{\kappa}=\mathbb{T}$. The forward graininess $\mu_{f}: \mathbb{T}_{\kappa} \rightarrow \mathbb{R}_{0}^{+}$is defined by

$$
\mu_{f}(t)=\sigma(t)-t .
$$

Definition 2.2. [7,22] For $x: \mathbb{T} \rightarrow R$ and $t \in \mathbb{T}^{\kappa}$, we define the " $\Delta$ " derivative of $x(t)$, $x^{\Delta}(t)$, to be the number (when it exists), with the property that, for any $\varepsilon>0$, there is neighborhood $U$ of $t$ such that

$$
\left|[x(\sigma(t))-x(s)]-x^{\Delta}(t)[\sigma(t)-s]\right|<\varepsilon|\sigma(t)-s|
$$

for all $s \in U$. For $x: \mathbb{T} \rightarrow R$ and $t \in \mathbb{T}_{\kappa}$, we define the " $\nabla$ " derivative of $x(t), x^{\Delta}(t)$, to be the number(when it exists), with the property that, for any $\varepsilon>0$, there is a neighborhood $V$ of $t$ such that

$$
\left|[x(\rho(t))-x(s)]-x^{\nabla}(t)[\rho(t)-s]\right|<\varepsilon|\rho(t)-s|
$$

for all $s \in V$.

Definition 2.3. [22] If $F^{\Delta}(t)=f(t)$, then we define the " $\Delta$ " integral by

$$
\int_{a}^{t} f(s) \Delta s=F(t)-F(a) .
$$

If $F^{\nabla}(t)=f(t)$, then we define the " $\nabla$ " integral by

$$
\int_{a}^{t} f(s) \nabla s=F(t)-F(a) .
$$

Lemma 2.1. [23] The following formulas hold:

(i) $\left(\int_{a}^{t} f(t) \Delta s\right)^{\Delta}=f(t)$,

(ii) $\left(\int_{a}^{t} f(t) \Delta s\right)^{\nabla}=f(\rho(t))$,

(iii) $\left(\int_{a}^{t} f(t) \nabla s\right)^{\Delta}=f(\sigma(t))$,

(iv) $\left(\int_{a}^{t} f(t) \nabla s\right)^{\nabla}=f(t)$.

Lemma 2.2. [7, Theorem 1.75 in p. 28] If $f \in C_{r d}$ and $t \in \mathbb{T}^{\kappa}$, then

$$
\int_{t}^{\sigma(t)} f(\tau) \Delta \tau=\mu_{f}(t) f(t) .
$$


According to [23, Theorem 1.30 in p. 9], we have the following lemma, which can be proved easily. Here, we omit it.

Lemma 2.3. Let $a, b \in \mathbb{T}$ and $f \in C_{l d}$.

(i) If $\mathbb{T}=\mathbb{R}$, then

$$
\int_{a}^{b} f(t) \nabla t=\int_{a}^{b} f(t) d t
$$

where the integral on the right is the usual Riemann integral from calculus.

(ii) If $[a, b]$ consists of only isolated points, then

$$
\int_{a}^{b} f(t) \nabla t=\left\{\begin{aligned}
\sum_{t \in(a, b]} \mu_{b}(t) f(t), & \text { if } a<b \\
0, & \text { if } a=b \\
-\sum_{t \in(a, b]} \mu_{b}(t) f(t), & \text { if } a>b .
\end{aligned}\right.
$$

(iii) If $\mathbb{T}=h \mathbb{Z}=\{h k: k \in \mathbb{Z}\}$, where $h>0$, then

$$
\int_{a}^{b} f(t) \nabla t=\left\{\begin{array}{c}
\sum_{k=\frac{a}{h}+1}^{\frac{b}{h}} f(k h) h, \quad \text { if } a<b, \\
-\sum_{k=\frac{b}{h}+1}^{\frac{a}{h}} f(k h) h, \text { if } a>b .
\end{array}\right.
$$

(iv) If $\mathbb{U}=\mathbb{Z}$, then

$$
\int_{a}^{b} f(t) \nabla t=\left\{\begin{aligned}
\sum_{t=a+1}^{b} f(t), & \text { if } a<b, \\
0, & \text { if } a=b, \\
-\sum_{t=b+1}^{a} f(t), & \text { if } a>b .
\end{aligned}\right.
$$

In what follows, we list the fixed point theorems that will be used in this article.

Theorem 2.4. [24] Let E be a Banach space and $P \subset E$ be a cone. Suppose $\Omega_{1}, \Omega_{2} \subset$ E open and bounded, $0 \in \Omega_{1} \subset \bar{\Omega}_{1} \subset \Omega_{2} \subset \bar{\Omega}_{2}$. Assume $A:\left(\bar{\Omega}_{2} \backslash \Omega_{1}\right) \cap P \rightarrow$ Pis completely continuous. If one of the following conditions holds

(i) $\|A x\| \leq\|x\|, \forall x \in \partial \Omega_{1} \cap P,\|A x\| \geq\|x\|, \forall x \in \partial \Omega_{2} \cap P$;

(ii) $\|A x\| \geq\|x\|, \forall x \in \partial \Omega_{1} \cap P,\|A x\| \leq\|x\|, \forall x \in \partial \Omega_{2} \cap P$.

Then, $A$ has a fixed point in $\left(\bar{\Omega}_{2} \backslash \Omega_{1}\right) \cap P$.

Theorem 2.5. [25] Let $P$ be a cone in the real Banach space E. Set

$$
P(\gamma, r)=\{u \in P, \gamma(u)<r\} .
$$

If $\alpha$ and $\gamma$ are increasing, nonnegative continuous functionals on $P$, let $\theta$ be a nonnegative continuous functional on $P$ with $\theta(0)=0$ such that for some positive constants $r, M$,

$$
\gamma(u) \leq \theta(u) \leq \alpha(u) \text { and }\|u\| \leq M \gamma(u) .
$$


for all $u \in \overline{P(\gamma, r)}$. Further, suppose there exists positive numbers $a<b<r$ such that

$$
\theta(\lambda u) \leq \lambda \theta(u) \text { for all } 0 \leq \lambda \leq 1, \quad u \in \partial P(\theta, b) .
$$

If $A: \overline{P(\gamma, r)} \rightarrow$ Pis completely continuous operator satisfying

(i) $\chi(A u)>r$ for all $u \in \partial P(\gamma, r)$;

(ii) $\theta(A u)<b$ for all $u \in \partial P(\theta, r)$;

(iii) $P(\alpha, b) \neq$ Dand $\alpha(A u)>a$ for all $u \in \partial P(\alpha, a)$.

Then, $A$ has at least two fixed points $u_{1}$ and $u_{2}$ such that

$$
a<\alpha\left(u_{1}\right) \text {, with } \theta\left(u_{1}\right)<b \text {, and } b<\theta\left(u_{2}\right) \text {, with } \gamma\left(u_{1}\right)<r \text {, }
$$

Let $a, b, c$ be constants, $P_{r}=\{u \in P:\|u\|<r\}, P(\psi, b, d)=\{u \in P: a \leq \psi(u),\|u\| \leq b\}$.

Theorem 2.6. [26] Let $A: \bar{P}_{c} \rightarrow \bar{P}_{c}$ be a completely continuous map and $\psi$ be a nonnegative continuous concave functional on $P$ such that for $\forall u \in \bar{P}_{c}$, there holds $\psi(u) \leq\|u\|$. Suppose there exist $a, b, d$ with $0<a<b<d \leq c$ such that

(i) $\{u \in P(\psi, b, d): \psi(u)>b\} \neq$ Dand $\psi(A u)>b$ for all $u \in P(\psi, b, d)$;

(ii) $\|A u\|<a$ for all $u \in \bar{P}_{a}$;

(iii) $\psi(A u)>b$ for all $u \in P(\psi, b, d)$ with $\|A u\|>d$.

Then, $A$ has at least three fixed points $u_{1}, u_{2}$, and $u_{3}$ satisfying

$$
\left\|u_{1}\right\|<a, b<\alpha\left(u_{2}\right),\left\|u_{3}\right\|>a, \text { and }\left\|u_{3}\right\|<b .
$$

Let the Banach space $E=C_{l d}[0, T]$ be endowed with the norm $\|u\|=\sup _{t \in[0, T]} u(t)$, and cone $P \subset E$ is defined as

$$
P=\left\{u \in E, u(t) \geq 0 \text { for } t \in[0, T] \text { and } u^{\Delta \nabla}(t) \leq 0 \text { for } t \in(0, T), u^{\Delta}(T)=0\right\} .
$$

It is obvious that $\|u\|=u(T)$ for $u \in P$. Define $A: P \rightarrow E$ as

$$
\begin{aligned}
(A u)(t)= & \int_{0}^{t} \phi_{q}\left(\int_{s}^{T} h(\tau) f(\tau, u(\tau)) \nabla \tau\right) \Delta s+\delta \phi_{q}\left(\int_{0}^{T} h(s) f(s, u(s)) \nabla s\right) \\
& +\sum_{i=1}^{m-2} \beta_{i} \phi_{q}\left(\int_{\xi_{i}}^{T} h(s) f(s, u(s)) \nabla s\right)
\end{aligned}
$$

for $t \in[0, T]$.

In what follows, we give the main lemmas which are important for getting the main results.

Lemma 2.7. $A: P \rightarrow P$ is completely continuous.

Proof. First, we try to prove that $A: P \rightarrow P$.

$$
(A u)^{\Delta}(t)=\phi_{q}\left(\int_{t}^{T} h(s) f(s, u(s)) \nabla s\right) .
$$

Thus, $(A u)^{\Delta}(T)=0$ and by Lemma 2.1 we have $(A u)^{\Delta \nabla}(t)=-h(t) f(t, u(t)) \leq 0$ for $t \in$ $(0, T)$. Consequently, $A: P \rightarrow P$.

By standard argument we can prove that $A$ is completely continuous. For more details, see [27]. The proof is complete.

Lemma 2.8. For $u \in P$, there holds $u(t) \geq \frac{t}{T}\|u\|$ for $t \in[0, T]$ for $t \in[0, T]$. 
Proof. For $u \in P$, we have $u^{\Delta \nabla}(t) \leq 0$, it follows that $u^{\Delta}(t)$ is non-increasing. Therefore, for $0<t<T$,

$$
u(t)-u(0)=\int_{0}^{t} u^{\Delta}(s) \Delta s \geq t u^{\Delta}(t)
$$

and

$$
u(T)-u(t)=\int_{t}^{T} u^{\Delta}(s) \Delta s \leq(T-t) u^{\Delta}(t)
$$

thus

$$
u(T)-u(0) \leq T u^{\Delta}(t) .
$$

Combining (2.1) and (2.3) we have

$$
T(u(t)-u(0)) \geq T t u^{\Delta}(t) \geq t(u(T)-u(0)),
$$

as $u(0) \geq 0$, it is immediate that

$$
u(t) \geq \frac{t u(T)+(T-t) u(0)}{T} \geq \frac{t}{T} u(T)=\frac{t}{T}\|u\| .
$$

The proof is complete.

\section{Existence of at least one positive solution}

First, we give some notations. Set

$$
\begin{aligned}
\Lambda & =\left(\delta+\sum_{i=1}^{m-2} \beta_{i}+T\right) \phi_{q}\left(\int_{0}^{T} h(s) \nabla s\right), \\
B & =\frac{\xi_{1}}{T}\left(\delta \phi_{q}\left(\int_{0}^{T} h(s) \nabla s\right)+\sum_{i=1}^{m-2} \beta_{i} \phi_{q}\left(\int_{\xi_{i}}^{T} h(s) \nabla s\right)+\int_{0}^{T} \phi_{q}\left(\int_{s}^{T} h(\tau) \nabla \tau\right) \Delta s\right) .
\end{aligned}
$$

Theorem 3.1. Assume in addition to $(S 1)$ and (S2), the following conditions are satisfied, there exists $0<r<\frac{\xi_{1} \rho}{T}<\rho<\infty$ such that

(H1) $f(t, u) \leq \phi_{p}\left(\frac{u}{\Lambda}\right)$, for $t \in[0, T], u \in[0, r]$;

(H2) $f(t, u) \geq \phi_{p}\left(\frac{u}{B}\right)$, for $t \in\left[\xi_{1}, T\right], u \in\left[\frac{\xi_{1} \rho}{T}, \rho\right]$.

Then, BVP (1.6) has at least one positive solution.

Proof. Cone $P$ is defined as above. By Lemma 2.7 we know that $A: P \rightarrow P$ is completely continuous. Set $\Omega_{r}=\{u \in E,\|u\|<r\}$. In view of (H1), for $u \in \partial \Omega_{r} \cap P$,

$$
\begin{aligned}
\|A u\|= & (A u)(T)=\delta \phi_{q}\left(\int_{0}^{T} h(s) f(s, u(s)) \nabla s\right)+\sum_{i=1}^{m-2} \beta_{i} \phi_{q}\left(\int_{\xi_{i}}^{T} h(s) f(s, u(s)) \nabla s\right) \\
& \quad+\int_{0}^{T} \phi_{q}\left(\int_{s}^{T} h(\tau) f(\tau, u(\tau)) \nabla \tau\right) \Delta s \\
\leq & \left(\delta+\sum_{i=1}^{m-2} \beta_{i}+T\right) \phi_{q}\left(\int_{0}^{T} \phi_{p}\left(\frac{u(s)}{\Lambda}\right) h(s) \nabla s\right) \\
\leq & \frac{\|u\|}{\Lambda}\left(\delta+\sum_{i=1}^{m-2} \beta_{i}+T\right) \phi_{q}\left(\int_{0}^{T} h(s) \nabla s\right) \leq\|u\|,
\end{aligned}
$$

which means that for $u \in \partial \Omega_{r} \cap P,\|A u\| \leq\|u\|$.

On the other hand, for $u \in P$, in view of Lemma 2.8, there holds $u(t) \geq \frac{\xi_{1}}{T}\|u\|$, for $t \in$ $\left[\xi_{1}, T\right]$. Denote $\Omega_{\rho}=\{u \in E,\|u\|<\rho\}$. Then for $u \in \partial \Omega_{\rho} \cap P$, considering (H2), we have 


$$
\begin{aligned}
\|A u\|=(A u)(T)= & \delta \phi_{q}\left(\int_{0}^{T} h(s) f(s, u(s)) \nabla s\right)+\sum_{i=1}^{m-2} \beta_{i} \phi_{q}\left(\int_{\xi_{i}}^{T} h(s) f(s, u(s)) \nabla s\right) \\
& +\int_{0}^{T} \phi_{q}\left(\int_{s}^{T} h(\tau) f(\tau, u(\tau)) \nabla \tau\right) \Delta s \\
\geq & \delta \phi_{q}\left(\int_{0}^{T} \phi_{p}\left(\frac{u(s)}{B}\right) h(s) \nabla s\right)+\sum_{i=1}^{m-2} \beta_{i} \phi_{q}\left(\int_{\xi_{i}}^{T} \phi_{p}\left(\frac{u(s)}{B}\right) h(s) \nabla s\right) \\
& +\int_{0}^{T} \phi_{q}\left(\int_{s}^{T} \phi_{p}\left(\frac{u(\tau)}{B}\right) h(\tau) \nabla \tau\right) \Delta s \\
\geq & \frac{\xi_{1}\|u\|}{T B}\left(\delta \phi_{q}\left(\int_{0}^{T} h(s) \nabla s\right)+\sum_{i=1}^{m-2} \beta_{i} \phi_{q}\left(\int_{\xi_{i}}^{T} h(s) \nabla s\right)+\int_{0}^{T} \phi_{q}\left(\int_{s}^{T} h(\tau) \nabla \tau\right) \Delta s\right) \\
= & \|u\| .
\end{aligned}
$$

which implies that for $u \in \partial \Omega_{\rho} \cap P,\|A u\| \geq\|u\|$ Therefore, the immediate result of Theorem 2.4 is that $A$ has at least one fixed point $u \in\left(\Omega_{\rho} \mid \Omega_{r}\right) \cap P$. Also, it is obvious that the fixed point of $A$ in cone $P$ is equivalent to the positive solution of BVP (1.6), this yields that BVP (1.6) has at least one positive solution $u$ satisfies $r \leq\|u\| \leq \rho$. The proof is complete.

Here is an example.

Example 3.2. Let $\mathbb{T}=\mathbb{P}_{1,1}=\bigcup_{k=0}^{\infty}[2 k, 2 k+1]$. Consider the following four point $B V P$ on time scale $\mathbb{P}_{1,1}$.

$$
\left\{\begin{array}{l}
x^{\Delta \nabla}(t)+f(t, u(t))=0, \quad t \in[0, T]_{\mathbb{T}} \\
x(0)-2 x^{\Delta}(0)=x^{\Delta}(2)+x^{\Delta}(3), \quad x^{\Delta}(4)=0,
\end{array}\right.
$$

where

$$
f(t, u)=\left\{\begin{array}{lr}
\frac{t u}{128}, & 0 \leq u \leq 100 \\
\frac{39 t}{512}(u-100)+\frac{25 t}{32}, & 00 \leq u \leq 500 \\
\frac{t u}{16}, & u \geq 500
\end{array}\right.
$$

and $h(t)=1, T=4, \xi_{1}=2, \xi_{2}=3, \delta=2, \beta_{1}=\beta_{2}=1, p=q=2$. In what follows, we try to calculate $\Lambda, B$. By Lemmas 2.2 and 2.3, we have

$$
\begin{gathered}
\Lambda=\left(\delta+\sum_{i=1}^{m-2} \beta_{i}+T\right) \phi_{q}\left(\int_{0}^{T} h(s) \nabla s\right) \\
=(2+1+1+4) \int_{0}^{4} \nabla s \\
=8 \times\left(\int_{0}^{1} d s+\int_{2}^{3} d s+\int_{1}^{2} \nabla s+\int_{3}^{4} \nabla s\right) \\
=8 \times\left(\int_{0}^{1} d s+\int_{2}^{3} d s+v(2) \times 1+v(4) \times 1\right) \\
=8 \times(1+1+1+1)=32 . \\
B=\frac{\xi}{T}\left(\delta \phi_{q}\left(\int_{0}^{T} h(s) \nabla s\right)+\sum_{i=1}^{m-2} \beta_{i} \phi_{q}\left(\int_{\xi_{i}}^{T} h(s) \nabla s\right)+\int_{0}^{T} \phi_{q}\left(\int_{s}^{T} h(\tau) \nabla \tau\right) \Delta s\right) \\
=\frac{2}{4}\left(2 \int_{0}^{4} \nabla s+\int_{2}^{4} \nabla s+\int_{3}^{4} \nabla s+\int_{0}^{4} \int_{s}^{4} \nabla \tau \Delta s\right) \\
=\frac{1}{2}\left(8+2+1+\int_{0}^{1} \int_{s}^{4} \nabla \tau \Delta s+\int_{1}^{2} \int_{2}^{4} \nabla \tau \Delta s+\int_{2}^{3} \int_{s}^{4} \nabla \tau \Delta s+\int_{3}^{4} \int_{s}^{4} \nabla \tau \Delta s\right) \\
=\frac{1}{2}\left(11+\int_{0}^{1} \int_{s}^{4} \nabla \tau \Delta s+\int_{1}^{2} \int_{s}^{4} \nabla \tau \Delta s+\int_{2}^{3} \int_{s}^{4} \nabla \tau \Delta s+\int_{3}^{4} \int_{s}^{4} \nabla \tau \Delta s\right),
\end{gathered}
$$


where

$$
\begin{aligned}
\int_{0}^{1} \int_{s}^{4} \nabla \tau \Delta s & =\int_{0}^{1}\left(\int_{s}^{1} \nabla \tau+\int_{1}^{4} \nabla \tau\right) \Delta s \\
& =\int_{0}^{1} \int_{s}^{1} d \tau d s+\int_{0}^{1}\left(\int_{1}^{2} \nabla \tau+\int_{2}^{3} \nabla \tau+\int_{3}^{4} \nabla \tau\right) \Delta s \\
& =\int_{0}^{1}\left(\int_{s}^{1} d \tau+\int_{2}^{3} d \tau\right) d s+\int_{0}^{1}\left(\int_{1}^{2} \nabla \tau+\int_{3}^{4} \nabla \tau\right) \Delta s \\
& =\frac{1}{2}+1+1+1=\frac{7}{2} \\
\int_{2}^{3} \int_{s}^{4} \nabla \tau \Delta s & =\int_{2}^{3}\left(\int_{s}^{3} \nabla \tau+\int_{3}^{4} \nabla \tau\right) \Delta s \\
& =\int_{2}^{3} \int_{s}^{3} d \tau d s+\int_{2}^{3} \int_{3}^{4} \nabla \tau \Delta s \\
& =\frac{1}{2}+1=\frac{3}{2}, \\
\int_{1}^{2} \int_{s}^{4} \nabla \tau \Delta s & =\sigma(1) \times \int_{1}^{4} \nabla \tau=3 \\
\int_{3}^{4} \int_{s}^{4} \nabla \tau \Delta s & =\sigma(3) \times \int_{3}^{4} \nabla \tau=1
\end{aligned}
$$

Thus, $B=\frac{1}{2}\left(11+\frac{7}{2}+\frac{3}{2}+3+1\right)=10$. Let $r=100<\frac{2}{4} \rho<\rho=1000$. Then, we have

(i) $f(t, u) \leq \frac{4 u}{128}=\frac{u}{32}=\phi_{p}\left(\frac{u}{\Lambda}\right)$, for $t \in[0,4], u \in[0,100]$;

(ii) $f(t, u) \geq \frac{2 u}{16}=\frac{u}{8}>\phi_{p}\left(\frac{u}{B}\right)$, for $t \in[2,4], u \in[500,1000]$.

Thus, if all the conditions in Theorem 3.1 satisfied, then BVP (3.1) has at least one positive solution lies between 100 and 1000.

\section{Existence of at least two positive solutions}

In this section, we will apply fixed point Theorem 2.5 to prove the existence of at least two positive solutions to the nonlinear BVP (1.6).

Fix $\eta \in \mathbb{T}$ such that

$$
0<\xi_{m-2} \leq \eta<T
$$

and define the increasing, nonnegative, continuous functionals $\gamma, \theta, \alpha$ on $P$ by

$$
\begin{aligned}
& \gamma(u)=\min _{t \in[\xi 1, \eta]} u(t)=u\left(\xi_{1}\right), \\
& \theta(u)=\max _{t \in\left[0, \xi_{m-2}\right]} u(t)=u\left(\xi_{m-2}\right), \\
& \alpha(u)=\min _{t \in[\eta, T]} u(t)=u(\eta) .
\end{aligned}
$$

We can see that, for $u L P$, there holds

$$
\gamma(u) \leq \theta(u) \leq \alpha(u) .
$$


In addition, Lemma 2.8 implies that $\gamma(u)=u\left(\xi_{1}\right) \geq \frac{\xi_{1}}{T}\|u\|$ which means that

$$
\|u\| \leq \frac{T}{\xi_{1}} \gamma(u) \text { for } u \in P .
$$

We also see that

$$
\theta(\lambda u)=\lambda \theta(u) \text { for } \lambda \in[0,1], u \in \partial P(\theta, b) .
$$

For convenience, we give some notations,

$$
\begin{aligned}
K & =\left(\delta+\sum_{i=1}^{m-2} \beta_{i}+\xi_{m-2}\right) \phi_{q}\left(\int_{0}^{T} h(s) \nabla s\right), \\
M & =\delta \phi_{q}\left(\int_{0}^{T} h(s) \nabla s\right)+\sum_{i=1}^{m-2} \beta_{i} \phi_{q}\left(\int_{\xi_{i}}^{T} h(s) \nabla s\right)+\int_{0}^{\xi_{1}} \phi_{q}\left(\int_{s}^{T} h(\tau) \nabla \tau\right) \Delta s, \\
L & =\delta \phi_{q}\left(\int_{0}^{T} h(s) \nabla s\right)+\sum_{i=1}^{m-2} \beta_{i} \phi_{q}\left(\int_{\xi_{i}}^{T} h(s) \nabla s\right)+\int_{0}^{\eta} \phi_{q}\left(\int_{s}^{T} h(\tau) \nabla \tau\right) \Delta s .
\end{aligned}
$$

Theorem 4.1. Assume in addition to $(S 1)$, (S2) there exist positive constants $a<\frac{T}{\eta} a<b<\frac{T}{\xi_{m-2}} b<$ csuch that the following conditions hold

(H3) $f(t, u)>\varphi_{p}(c / M)$ for $t \in\left[\xi_{1}, T\right] u \in\left[c, T c / \xi_{1}\right]$;

(H4) $f(t, u)<\varphi_{p}(b / K)$ for $t \in\left[0, \xi_{m-2}\right], u \in\left[b, T b / \xi_{m-2}\right]$;

(H5) $f(t, u)>\varphi_{p}(a / L)$ for $t \in[\eta, T], u \in[a, T a / \eta]$.

Then BVP (1.6) has at least two positive solutions $u_{1}$ and $u_{2}$ such that

$$
\alpha\left(u_{1}\right)>a, \text { with } \theta\left(u_{1}\right)<b \text {, and } b<\theta\left(u_{2}\right) \text {, with } \gamma\left(u_{2}\right)<c \text {. }
$$

Proof. From Lemma 2.7 we know that $A: P(\gamma, c) \rightarrow P$ is completely continuous. In what follows, we will prove the result step by step.

Step one: To verify $(i)$ of theorem 2.5 holds.

We choose $u \in \partial P(\gamma, c)$, then $\gamma(u)=\min _{t \in\left[\xi_{1}, \eta\right]} u(t)=u\left(\xi_{1}\right)=c$. This implies that $u(t)$ $\geq c$ for $t \in\left[\xi_{1}, T\right]$, considering that $\|u\| \leq \frac{T}{\xi_{1}} \gamma(u)=\frac{T}{\xi_{1}} c$, we have

$$
c \leq u(t) \leq \frac{T}{\xi_{1}} c \text { for } t \in\left[\xi_{1}, T\right] .
$$

As a consequence of (H3),

$$
f(t, u(t))>\phi_{p}\left(\frac{c}{M}\right) \text { for } t \in\left[\xi_{1}, T\right] .
$$

Since $A u \in P$, we have

$$
\begin{aligned}
\gamma(A u)=(A u)\left(\xi_{1}\right)= & \delta \phi_{q}\left(\int_{0}^{T} h(s) f(s, u(s)) \nabla s\right)+\sum_{i=1}^{m-2} \beta_{i} \phi_{q}\left(\int_{\xi_{i}}^{T} h(s) f(s, u(s)) \nabla s\right) \\
& +\int_{0}^{\xi_{1}} \phi_{q}\left(\int_{s}^{T} h(\tau) f(\tau, u(\tau)) \nabla \tau\right) \Delta s \\
& >\frac{c}{M}\left(\delta \phi_{q}\left(\int_{0}^{T} h(s) \nabla s\right)+\sum_{i=1}^{m-2} \beta_{i} \phi_{q}\left(\int_{\xi_{i}}^{T} h(s) \nabla s\right)+\int_{0}^{\xi_{1}} \phi_{q}\left(\int_{s}^{T} h(\tau) \nabla \tau\right) \Delta s\right) \\
& =c .
\end{aligned}
$$

Thus, (i) of Theorem 2.5 is satisfied. 
Step two: To verify $(i i)$ of Theorem 2.5 holds.

Let $u \in \partial P(\theta, b)$, then $\theta(u)=\max _{t \in\left[0, \xi_{m-2}\right]}=u\left(\xi_{m-2}\right)=b$, this implies that $0 \leq u(t) \leq b$, $t \in\left[0, \xi_{m-2}\right]$ and since $u \in P$, we have $\|u\|=u(T)$, note that $\|u\| \leq \frac{T}{\xi_{m-2}} u\left(\xi_{m-2}\right)=\frac{T}{\xi_{m-2}} \theta(u)=\frac{T}{\xi_{m-2}} b$. So,

$$
0 \leq u(t) \frac{T}{\xi_{m-2}} b \text { for } t \in[0, T] \text {. }
$$

From (H4) we know that $f(t, u(t))<\phi_{p}\left(\frac{b}{K}\right)$ for $t \in\left[0, \xi_{m-2}\right]$ and so

$$
\begin{aligned}
\theta(A u)=(A u)\left(\xi_{m-2}\right)= & \delta \phi_{q}\left(\int_{0}^{T} h(s) f(s, u(s)) \nabla s\right)+\sum_{i=1}^{m-2} \beta_{i} \phi_{q}\left(\int_{\xi_{i}}^{T} h(s) f(s, u(s)) \nabla s\right) \\
& +\int_{0}^{\xi_{m-2}} \phi_{q}\left(\int_{s}^{T} h(\tau) f(\tau, u(\tau)) \nabla \tau\right) \Delta s \\
& <\frac{b}{K}\left(\delta \phi_{q}\left(\int_{0}^{T} h(s) \nabla s\right)+\sum_{i=1}^{m-2} \beta_{i} \phi_{q}\left(\int_{\xi_{j}}^{T} h(s) \nabla s\right)\right. \\
& \left.+\int_{0}^{\xi_{m-2}} \phi_{q}\left(\int_{s}^{T} h(\tau) \nabla \tau\right) \Delta s\right) \\
& <\frac{b}{K}\left(\delta+\sum_{i=1}^{m-2} \beta_{i}+\xi_{m-2}\right) \phi_{q}\left(\int_{0}^{T} h(s) \nabla s\right)=b .
\end{aligned}
$$

Thus, (ii) of Theorem 2.5 holds.

Step three: To verify (iii) of Theorem 2.5 holds.

Choose $u_{0}(t)=\frac{a}{2}, t \in[0, T]$, obviously, $u_{0}(t) \in P(\alpha, a)$ and $\alpha\left(u_{0}\right)=\frac{a}{2}<a$, thus $P(\alpha, a) \neq \emptyset$.

Now, let $u \in \partial P(\alpha, a)$, then, $\alpha(u)=\min _{t L[\eta, T]} u(t)=u(\eta)=a$. Recalling that $\|u\| \leq \frac{T}{\eta} u(\eta)=\frac{T}{\eta} \alpha(u)=\frac{T}{\eta} a$. Thus, we have

$$
a \leq u(t) \leq \frac{T}{\eta} a \text { for } t \in[\eta, T] .
$$

From assumption (H5) we know that

$$
f(t, u(t))>\phi_{p}\left(\frac{a}{L}\right) \text { for } t \in[\eta, T]
$$

and so

$$
\begin{aligned}
\alpha(A u)=(A u)(\eta)= & \delta \phi_{q}\left(\int_{0}^{T} h(s) f(s, u(s)) \nabla s\right)+\sum_{i=1}^{m-2} \beta_{i} \phi_{q}\left(\int_{\xi_{i}}^{T} h(s) f(s, u(s)) \nabla s\right) \\
& +\int_{0}^{\eta} \phi_{q}\left(\int_{s}^{T} h(\tau) f(\tau, u(\tau)) \nabla \tau\right) \Delta s \\
& >\frac{a}{L}\left(\delta \phi_{q}\left(\int_{0}^{T} h(s) \nabla s\right)+\sum_{i=1}^{m-2} \beta_{i} \phi_{q}\left(\int_{\xi_{i}}^{T} h(s) \nabla s\right)+\int_{0}^{\eta} \phi_{q}\left(\int_{s}^{T} h(\tau) \nabla \tau\right) \Delta s\right) \\
& =a .
\end{aligned}
$$

Therefore, all the conditions of Theorem 2.5 are satisfied, thus $A$ has at least two fixed points in $P(\gamma, c)$, which implies that BVP (1.6) has at least two positive solutions $u_{1}, u_{2}$ which satisfies (4.1). The proof is complete. 
Example 4.2. Let $\mathbb{T}=\left\{2^{n}, n \in \mathbb{Z}\right\} \cup\{0\}$. Consider the following four point boundary value problem on time scale $\mathbb{T}$.

$$
\left\{\begin{array}{l}
\left(\phi_{p}\left(x^{\Delta}\right)\right)^{\nabla}(t)+t f(t, u(t))=0, \quad t \in[0,8]_{\mathbb{T}}, \\
x(0)-x^{\Delta}(0)=x^{\Delta}(1)+2 x^{\Delta}(2), x^{\Delta}(8)=0
\end{array}\right.
$$

where

$$
f(t, u)=\left\{\begin{array}{lr}
|\sin t|+\frac{u}{10^{5}}, & 0 \leq u \leq 9.3 \times 10^{6}, \\
|\sin t|+93, & 9.3 \times 10^{6} \leq u \leq 4 \times 10^{8}, \\
|\sin t|+\frac{247 u}{6 \times 10^{8}}-\frac{215}{3}, & u \geq 4 \times 10^{8},
\end{array}\right.
$$

and $h(t)=t, T=8, \xi_{1}=1, \xi_{2}=2, \delta=1, \beta_{1}=1, \beta_{2}=2, p=3 / 2, q=3$. In what follows, we try to calculate $K, M$, L. By Lemmas 2.2 and 2.3, we have

$$
\begin{aligned}
K= & \left(\delta+\sum_{i=1}^{m-2} \beta_{i}+\xi_{m-2}\right) \phi_{q}\left(\int_{0}^{T} h(s) \nabla s\right) \\
= & (1+1+2+2) \phi_{q}\left(\int_{0}^{8} s \nabla s\right) \\
= & 6 \times\left(\int_{0}^{1} s \nabla s+\int_{1}^{2} s \nabla s+\int_{2}^{4} s \nabla s+\int_{4}^{8} s \nabla s\right)^{2} \\
= & 6 \times(v(1) \times 1+v(2) \times 2+v(4) \times 4+v(8) \times 8)^{2} \\
= & 6 \times(1+2+8+32)^{2}=6 \times 1849=11094 . \\
M= & \delta \phi_{q}\left(\int_{0}^{T} h(s) \nabla s\right)+\sum_{i=1}^{m-2} \beta_{i} \phi_{q}\left(\int_{\xi_{i}}^{T} h(s) \nabla s\right)+\int_{0}^{\xi_{i}} \phi_{q}\left(\int_{s}^{T} h(\tau) \nabla \tau\right) \Delta s \\
= & \left(\int_{0}^{8} s \nabla s\right)^{2}+\left(\int_{1}^{8} s \nabla s\right)^{2}+2\left(\int_{2}^{8} s \nabla s\right)^{2}+\int_{0}^{1} \phi_{q}\left(\int_{s}^{8} \tau \nabla \tau\right) \Delta s \\
= & (1+2+8+32)^{2}+(2+8+32)^{2}+2 \times(8+32)^{2}+\left(\int_{0}^{8} s \nabla s\right)^{2} \\
= & 2 *(1+2+8+32)^{2}+(2+8+32)^{2}+2 \times(8+32)^{2}=8662 . \\
L= & \delta \phi_{q}\left(\int_{0}^{T} h(s) \nabla s\right)+\sum_{i=1}^{m-2} \beta_{i} \phi_{q}\left(\int_{\xi_{i}}^{T} h(s) \nabla s\right)+\int_{0}^{\eta} \phi_{q}\left(\int_{s}^{T} h(\tau) \nabla \tau\right) \Delta s \\
= & \left(\int_{0}^{8} s \nabla s\right)^{2}+\left(\int_{1}^{8} s \nabla s\right)^{2}+2\left(\int_{2}^{8} s \nabla s\right)^{2}+\int_{0}^{4}\left(\int_{s}^{8} h(\tau) \nabla \tau\right)^{2} \Delta s \\
= & (1+2+8+32)^{2}+(2+8+32)^{2}+2 \times(8+32)^{2} \\
& +\int_{0}^{1}\left(\int_{s}^{8} h(\tau) \nabla \tau\right)^{2} \Delta s+\int_{1}^{2}\left(\int_{s}^{8} h(\tau) \nabla \tau\right)^{2} \Delta s+\int_{2}^{4}\left(\int_{s}^{8} h(\tau) \nabla \tau\right)^{2} \Delta s \\
= & 6813+\mu(0) \times\left(\int_{0}^{8} s \nabla s\right)^{2}+\mu(1) \times\left(\int_{0}^{8} s \nabla s\right)^{2}+\mu(2) \times\left(\int_{0}^{8} s \nabla s\right)^{2} \\
= & 6813+(1+2+8+32)^{2}+(2+8+32)^{2}+2 \times(8+32)^{2}=13626 . \\
& \\
& \\
&
\end{aligned}
$$

Let $a=10^{6}, b=10^{8}, c=10^{9}$, then we have

(i) $f(t, u) \geq 340>\left(\frac{10^{9}}{8662}\right)^{1 / 2}=\phi_{p}\left(\frac{c}{M}\right)$, for $t \in[1,8], u \in\left[10^{9}, 8 \times 10^{9}\right]$;

(ii) $f(t, u) \leq 94<\left(\frac{10^{8}}{11094}\right)^{1 / 2}=\phi_{p}\left(\frac{b}{K}\right)$, for $t \in[0,2], u \in\left[10^{8}, 4 \times 10^{8}\right]$; 
(iii) $f(t, u)>9>\left(\frac{10^{6}}{13326}\right)^{1 / 2}=\phi_{p}\left(\frac{a}{L}\right)$, for $t \in[4,8], u \in\left[10^{6}, 2 \times 10^{6}\right]$.

Thus, if all the conditions in Theorem 4.1 are satisfied, then BVP (4.2) has at least two positive solutions satisfying (4.1).

\section{Existence of at least three positive solutions}

Let $\psi(u)=\min _{t \in\left[\xi_{1}, T\right]} u(t)$, then $0<\psi(u) \leq\|u\|$. Denote

$$
\begin{aligned}
& D=\delta \phi_{q}\left(\int_{0}^{T} h(s) \nabla s\right)+\sum_{i=1}^{m-2} \beta_{i} \phi_{q}\left(\int_{\xi_{i}}^{T} h(s) \nabla s\right)+\int_{0}^{\xi_{1}} \phi_{q}\left(\int_{s}^{T} h(\tau) \nabla \tau\right) \Delta s, \\
& R=\left(\delta+\sum_{i=1}^{m-2} \beta_{i}+T\right) \phi_{q}\left(\int_{0}^{T} h(s) \nabla s\right) .
\end{aligned}
$$

In this section, we will use fixed point Theorem 2.6 to get the existence of at least three positive solutions.

Theorem 5.1. Assume that there exists positive number $d, v, g$ satisfying $d<v<\min \left\{\frac{\xi_{1}}{T}, \frac{D}{R}\right\} g<g$, such that the following conditions hold.

(H6) $f(t, u)<\varphi_{p}(d / R), t \in[0, T], u \in[0, d]$;

(H7) $f(t, u)>\varphi_{p}(v / D), t \in\left[\xi_{1}, T\right], u \in\left[v, T v / \xi_{1}\right]$;

(H8) $f(t, u) \leq \varphi_{p}(g / R), t \in[0, T], u \in[0, g]$,

then $B V P(1.6)$ has at least three positive solutions $u_{1}, u_{2}$, $u_{3}$ satisfying

$$
\begin{aligned}
& \left\|u_{1}\right\|<d, \quad \psi\left(u_{2}\right)>v, \\
& \left\|u_{3}\right\|>d, \quad \text { with } \psi\left(u_{3}\right)<v .
\end{aligned}
$$

Proof. From Lemma 2.8 we know that $A: P \rightarrow P$ is completely continuous. Now we only need to show that all the conditions in Theorem 2.6 are satisfied.

For $u \in \bar{P}_{g^{\prime}}\|u\| \leq g$. By (H8), one has

$$
\begin{aligned}
\|A u\|=(A u)(T)= & \delta \phi_{q}\left(\int_{0}^{T} h(s) f(s, u(s)) \nabla s\right)+\sum_{i=1}^{m-2} \beta_{i} \phi_{q}\left(\int_{\xi_{i}}^{T} h(s) f(s, u(s)) \nabla s\right) \\
& +\int_{0}^{T} \phi_{q}\left(\int_{s}^{T} h(\tau) f(\tau, u(\tau)) \nabla \tau\right) \Delta s \\
& \leq \frac{g}{R}\left(\delta+\sum_{i=1}^{m-2} \beta_{i}+T\right) \phi_{q}\left(\int_{0}^{T} h(s) \nabla s\right) \\
& =g .
\end{aligned}
$$

Thus, $A: \bar{P}_{g} \rightarrow \bar{P}_{g}$. Similarly, by (H6), we can prove (ii) of Theorem 2.6 is satisfied. In what follows, we try to prove that $(i)$ of theorem 2.6 holds. Choose $u_{1}(t)=\frac{T}{\xi_{1}} v, t \in[0, T]$, obviously, $\psi\left(u_{1}\right)>v$, thus $\left\{u \in P\left(\psi, v, T v / \xi_{1}\right): \psi(u)>v\right\} \neq \emptyset$. For $u \in P\left(\psi, v, T v / \xi_{1}\right)$, 


$$
\begin{aligned}
\psi(A u)=(A u)\left(\xi_{1}\right)= & \delta \phi_{q}\left(\int_{0}^{T} h(s) f(s, u(s)) \nabla s\right)+\sum_{i=1}^{m-2} \beta_{i} \phi_{q}\left(\int_{\xi_{i}}^{T} h(s) f(s, u(s)) \nabla s\right) \\
& +\int_{0}^{\xi_{1}} \phi_{q}\left(\int_{s}^{T} h(\tau) f(\tau, u(\tau)) \nabla \tau\right) \Delta s \\
& >\frac{v}{D}\left(\delta \phi_{q}\left(\int_{0}^{T} h(s) \nabla s\right)+\sum_{i=1}^{m-2} \beta_{i} \phi_{q}\left(\int_{\xi_{i}}^{T} h(s) \nabla s\right)+\int_{0}^{\xi_{1}} \phi_{q}\left(\int_{s}^{T} h(\tau) \nabla \tau\right) \Delta s\right) \\
& =v .
\end{aligned}
$$

It remains to prove $(\mathrm{iii})$ of Theorem 2.6 holds. For $u \in P\left(\psi, v, T v / \xi_{1}\right)$, with $\|A u\|$ $>T v / \xi_{1}$, in view of Lemma 2.8 , there holds $\psi(A u)-(A u)\left(\xi_{1}\right) \geq \frac{\xi_{1}}{T}\|A u\|>v$, which implies that (iii) of Theorem 2.6 holds.

Therefore, all the conditions in Theorem 2.6 are satisfied. Thus, BVP (1.6) has at least three positive solutions satisfying (5.1). The proof is complete.

Example 5.2. Let $\mathbb{T}=[0,1] \cup \mathbb{N}$. Consider the following four point boundary value problem on time scale $\mathbb{T}$.

$$
\left\{\begin{array}{l}
\left(\phi_{p}\left(x^{\Delta}\right)\right)^{\nabla}(t)+e^{t}(t, u(t))=0, \quad t \in[0, T]_{\mathbb{\Psi}}, \\
x(0)-3 x^{\Delta}(0)=2 x^{\Delta}(1 / 2)+3 x^{\Delta}(1), \quad x^{\Delta}(8)=0,
\end{array}\right.
$$

where

$$
f(t, u)=\left\{\begin{array}{lc}
\frac{t}{20}+\left(\frac{u^{2}}{840}\right)^{3}, & 0 \leq u \leq 126 \\
\frac{t}{20}+18.9^{3}, & u \geq 126
\end{array}\right.
$$

and $h(t)=e^{t}, T=2, \xi_{1}=1 / 2, \xi_{2}=1, \delta=3, \beta_{1}=2, \beta_{2}=3, p=4, q=4 / 3$. In what follows, we try to calculate $D, R$. By Lemmas 2.2 and 2.3, we have

$$
\begin{aligned}
D= & \delta \phi_{q}\left(\int_{0}^{T} h(s) \nabla s\right)+\sum_{i=1}^{m-2} \beta_{i} \phi_{q}\left(\int_{\xi_{i}}^{T} h(s) \nabla s\right)+\int_{0}^{\xi_{1}} \phi_{q}\left(\int_{s}^{T} h(\tau) \nabla \tau\right) \Delta s \\
= & 3\left(\int_{0}^{2} e^{s} \nabla s\right)^{1 / 3}+2\left(\int_{1 / 2}^{2} e^{s} \nabla s\right)^{1 / 3}+3\left(\int_{1}^{2} e^{s} \nabla s\right)^{1 / 3}+\int_{s}^{1 / 2}\left(\int_{s}^{2} e^{\tau} \nabla \tau\right)^{1 / 3} \Delta s \\
= & 3\left(\int_{0}^{1} e^{s} d s+\int_{1}^{2} e^{s} \nabla s\right)^{1 / 3}+2\left(\int_{1 / 2}^{1} e^{s} d s+\int_{1}^{2} e^{s} \nabla s\right)^{1 / 3}+3\left(\int_{1}^{2} e^{s} \nabla s\right)^{1 / 3} \\
& +\int_{0}^{1 / 2}\left(\int_{s}^{1} e^{\tau} d \tau+\int_{1}^{2} e^{\tau} \nabla \tau\right)^{1 / 2} \Delta s \\
= & 3\left(e+e^{2}-1\right)^{1 / 3}+2\left(e+e^{2}-e^{1 / 2}\right)^{1 / 3}+3 e^{2 / 3}+\frac{3}{4}\left(e+e^{2}-1\right)^{4 / 3}-\frac{3}{4}\left(e+e^{2}-e^{1 / 2}\right)^{4 / 3} \\
\approx & 17.5216 . \\
R= & \left(\delta+\sum_{i=1}^{m-2} \beta_{i}+T\right) \phi_{q}\left(\int_{0}^{T} h(s) \nabla s\right)=(3+2+3+2)\left(\int_{0}^{2} e^{s} \nabla s\right)^{1 / 3} \\
= & 10\left(e+e^{2}-1\right)^{1 / 3}=20.8832 .
\end{aligned}
$$

Let $d=40, v=50, g=400$, then we have

(i) $f(t, u)<7.027=(40 / 20.8832)^{3}=\varphi_{p}(d / R)$, for $t \in[0,2], u \in[0,40]$;

(ii) $f(t, u)>23.2375=(50 / 17.5216)^{3}=\varphi_{p}(v / D)$, for $t \in[1 / 2,2], u \in[50,200]$;

(iii) $f(t, u)<7027.305=(400 / 20.8832)^{3}=\varphi_{p}(g / R)$, for $t \in[0,2], u \in[0,400]$. 
Thus, if all the conditions in Theorem 5.1 are satisfied, then BVP (5.2) has at least three positive solutions satisfying (5.1).

\section{Acknowledgements}

The authors were very grateful to the anonymous referee whose careful reading of the manuscript and valuable comments enhanced presentation of the manuscript. The study was supported by Pre-research project and Excellent Teachers project of the Fundamental Research Funds for the Central Universities (2011YYL079, 2011YXL047),

\section{Author details}

${ }^{1}$ School of Science, China University of Geosciences, Beijing 100083, P.R. China ${ }^{2}$ Department of Mathematics, Beijing Institute of Technology, Beijing 100081, P.R. China

\section{Authors' contributions}

WG and HL conceived of the study, and participated in its coordination. JZ drafted the manuscript. All authors read and approved the final manuscript.

\section{Competing interests}

The authors declare that they have no competing interests.

Received: 4 May 2011 Accepted: 17 January 2012 Published: 17 January 2012

\section{References}

1. Hilger, S: Analysis on measure chains-a unified approach to continuous and discrete calculus. Results Math. 18, 18-56 (1990)

2. Agarwal, RP, Bohner, M, Li, WT: Nonoscillation and Oscillation Theory for Functional Differential Equations. Pure and Applied Mathematics Series. Dekker, FL (2004)

3. Moshinsky, M: Sobre los problemas de condiciones a la frontiera en una dimension de caracteristicas discontinuas. Bol Soc Mat Mexicana. 7, 10-25 (1950)

4. Timoshenko, S: Theory of Elastic Stability. McGraw-Hill, New York (1961)

5. Zou, Y, Hu, Q, Zhang, R: On numerical studies of multi-point boundary value problem and its fold bifurcation. Appl Math Comput. 185, 527-537 (2007). doi:10.1016/j.amc.2006.07.064

6. Il'in, VA, Moiseev, El: Nonlocal boundary value problem of the second kind for a Sturm-Liouville operator. Diff Equ. 23 , 979-987 (1987)

7. Agarwal, RP, Bohner, M: Basic calculus on time scales and some of its applications. Results Math. 35, 3-22 (1999)

8. Agarwal, RP, O'Regan, D: Nonlinear boundary value problems on time scales. Nonlinear Anal. 44, 527-535 (2001). doi:10.1016/S0362-546X(99)00290-4

9. Anderson, D: Solutions to second-order three-point problems on time scales. J Diff Equ Appl. 8, 673-688 (2002). doi:10.1080/1023619021000000717

10. Kaufmann, ER: Positive solutions of a three-point boundary value problem on a time scale. Electron J Diff Equ. 82, 1-11 (2003)

11. Chyan, CJ, Henderson, J: Twin solutions of boundary value problems for differential equations on measure chains. J Comput Appl Math. 141, 123-131 (2002). doi:10.1016/S0377-0427(01)00440-X

12. Agarwal, RP, Lü, HSh, O'Regan, D: Eigenvalues and the one-dimensional p-Laplacian. J Math Anal Appl. 266, 383-400 (2002). doi:10.1006/jmaa.2001.7742

13. Sun, H, Tang, L, Wang, Y: Eigenvalue problem for $p$-Laplacian three-point boundary value problem on time scales. J Math Anal Appl. 331, 248-262 (2007). doi:10.1016/j.jmaa.2006.08.080

14. Geng, F, Zhu, D: Multiple results of p-Laplacian dynamic equations on time scales. Appl Math Comput. 193, 311-320 (2007). doi:10.1016/j.amc.2007.03.069

15. He, Z, Jiang, $X$ : Triple positive solutions of boundary value problems for $p$-Laplacian dynamic equations on time scales. J Math Anal Appl. 321, 911-920 (2006). doi:10.1016/j.jmaa.2005.08.090

16. Hong, S: Triple positive solutions of three-point boundary value problems for $p$-Laplacian dynamic equations. J Comput Appl Math. 206, 967-976 (2007). doi:10.1016/j.cam.2006.09.002

17. Graef, J, Kong, L: First-order singular boundary value problems with $p$-Laplacian on time scales. J Diff Equ Appl. 17, 831-839 (2011). doi:10.1080/10236190903443111

18. Anderson, DR: Existence of solutions for a first-order p-Laplacian BVP on time scales. Nonlinear Anal. 69, 4521-4525 (2008). doi:10.1016/j.na.2007.11.008

19. Goodrich, CS: Existence of a positive solution to a first-order p-Laplacian BVP on a time scale. Nonlinear Anal. $\mathbf{7 4}$ 1926-1936 (2011). doi:10.1016/j.na.2010.10.062

20. He, ZM: Double positive solutions of boundary value problems for $p$-Laplacian dynamic equations on time scales. Appl Anal. 84, 377-390 (2005). doi:10.1080/00036810500047956

21. Anderson, DR: Twin n-point boundary value problem. Appl Math Lett. 17, 1053-1059 (2004). doi:10.1016/j. aml.2004.07.008

22. Bohner, M, Peterson, A: Advances in Dynamic Equations on Time Scales. Birkhauser, Boston (2003)

23. Bohner, M, Peterson, A: Dynamic Equations on Time Scales. An Introduction with Applications. Birkhauser, Boston (2001)

24. Guo, D, Lakshmikantham, V: Nonlinear Problems in Abstract Cones. Academic Press, San Diego (1988)

25. Avery, Rl, Henderson, J: Two positive fixed points of nonlinear operators on ordered Banach spaces. Comm Appl Nonlinear Anal. 8, 27-36 (2001)

26. Leggett, RW, Williams, LR: Multiple positive fixed points of nonlinear operators on ordered Banach spaces. Indiana Univ Math J. 28, 673-688 (1979). doi:10.1512/iumj.1979.28.28046 
27. Zhao, J: Nonlocal boundary value problems of ordinary differential equations and dynamical equations on time scales. Doctoral thesis. Beijing Institute of Technology (2009)

doi:10.1186/1687-2770-2012-4

Cite this article as: Zhao et al:: Existence of positive solutions for nonlinear $m$-point boundary value problems on time scales. Boundary Value Problems 2012 2012:4.

Submit your manuscript to a SpringerOpen ${ }^{\odot}$ journal and benefit from:

- Convenient online submission

- Rigorous peer review

- Immediate publication on acceptance

- Open access: articles freely available online

- High visibility within the field

- Retaining the copyright to your article

Submit your next manuscript at $\boldsymbol{\nabla}$ springeropen.com 